\title{
Le Traitement Antibiotique Dans La Pédiatrie
}

Stoyan Papanov, (PhD)

Université médicale Plovdiv, Faculté de pharmacie, Département de pharmacognosie et de la chimie pharmaceutique, Bulgarie

Associate prof. Ekaterina Petkova, (PhD)

Université médicale Plovdiv, Medical College, Bulgarie

Prof. Zlatka Dimitrova, (PhD)

Université de Sofia St. Kliment Ohridski"-

Département de chimie et de pharmacie, Bulgarie

Vania Georgieva

Hôpital Universitaire Sofiamed, Bulgarie

Kalin Ivanov, (PhD)

Stanislava Ivanova, (PhD)

Prof. Rumen Mladenov, (PhD)

Université médicale Plovdiv, Faculté de pharmacie, Département de pharmacognosie et de la chimie pharmaceutique, Bulgarie

doi: 10.19044/esj.2016.v12n33p103 URL:http://dx.doi.org/10.19044/esj.2016.v12n33p103

Abstract

The current study should be paid extra attention because antibiotics are main cluster of drugs for treatment of child infections and illnesses. They are the most commonly prescribed medicines for children, relatively expensive and consume $60-70 \%$ of the budget for treatment of child illnesses, with exclusion only in malign sickness.

OBJECT: The current study represents common classification of antibiotics and the most commonly used antimicrobial agents during childhood.

RESEARCH METHODS

- Systematic approach and critical analysis of the available scientific periodicals

- Interview method of the survey (survey data pediatricians and general practitioners in the region of Plovdiv, Bulgaria.)

- Own research on the issue.

RESULTS AND DISCUSSION: Most often, doctors use:

Broad-spectrum penicillins: Amoxicillin, Amopen, Ospamox, Duomox

First generation cephalosporins: Ospexin, Cephalexin

Second-generation cephalosporins: Cefaclor

Third generation cephalosporins: Abricef, Cefotaxime, Rocephin, Fortum 
Fourth generation cephalosporins: Maxipime

Macrolides: Macropen

Aminoglycosides: Gentamicin, Amicacine

CONCLUSION: No significant differences are observed in the prescribed by the general practitioners and pediatricians antibiotics.

Keywords: Children, antibiotics, doctors, pharmacists

\section{Abstract}

Ce développement mérite une attention parce que les antibiotiques sont une classe majeure des agents pour traiter les maladies infectieuses chez les enfants. Ils sont les médicaments les plus couramment prescrits en pédiatrie, relativement coûteux et occupent 60-70\% du coût de traitement des maladies infantiles à l'exception de maligne.

BUT : Ce développement présente classification commune des antibiotiques et le plus couramment utilisés agents antimicrobiens dans l' enfance.

MÉTHODES DE RECHERCHE

- approche systématique et analyse critique des périodiques scientifiques disponibles

- méthode interview de l'enquête (données d'enquête de pédiatres et de médecins généralistes dans la région de Plovdiv Bulgarie.)

- propres recherches sur la question

RÉSULTATS ET DISCUSSION: Le plus souvent, les médecins utilisent:

Pénicillines à large spectre: Amoxicillin, Amopen, Ospamox, Duomox

Céphalosporines de première génération: Ospexin, Cephalexin

Céphalosporines de deuxième génération: Cefaclor

Céphalosporines de troisième génération: Abricef, Cefotaxime, Rocephin, Fortum

Céphalosporines de quatrième génération: Maxipime

Macrolides: Macropen

Aminoglicosides: Gentamicine, Amicacine

CONCLUSION: Aucune différence significative dans la prescription d'antibiotiques par les médecins généralistes et les pédiatres.

Mots-clefs: Enfants, antibiotiques, médecins, pharmaciens

\section{Introduction}

Ce développement mérite une attention parce que les antibiotiques sont une classe majeure des agents pour traiter les maladies infectieuses chez les enfants. Ils sont les médicaments les plus couramment prescrits en pédiatrie, relativement coûteux et occupent 60-70\% du coût de traitement des maladies infantiles à l'exception de maligne. Ce n'est pas sans importance, 
c'est le fait que les antibiotiques sont souvent prescrits ne suffit pas à justifier le traitement et la prévention de l'enfant. Par-dessus tout il doit y avoir des preuves de traitement. Très important est le fait que le médecin doit savoir quand et à quoi s'attendre d'un traitement avec des antibiotiques.

\section{But}

Ce développement présente classification commune des antibiotiques et le plus couramment utilisés agents antimicrobiens dans l' enfance.

\section{Matériel et méthodes}

L'objet de l'étude est l'utilisation d'antibiotiques dans l'enfance.

L'impact des sociétés pharmaceutiques.

Le reflét des effets indésirables et leur enregistrement.

\section{Sources D'information}

- littéraire

- données d'enquête de pédiatres et de médecins généralistes dans la région de Plovdiv Bulgarie.

La durée de l'étude porte sur la période du 1.05.2015 au 30.05.2016

Lieu d'étude sont les institutions médicales (soins hospitaliers et ambulatoires) de la région de Plovdiv Bulgarie.

\section{Méthodes de recherche}

- approche systématique et analyse critique des périodiques scientifiques disponibles

- méthode interview de l'enquête

- propres recherches sur la question

\section{Résultats et discussion}

Dans cette enquête prennent participation 44 medecins-23 pédiatres et 21 medecins généralistes.32 pharmaciens de maître et 28 pharmaciens adjoints

Parmi les répondants, 31 étaient des femmes et 13 hommes.La plupart ont acquis leur formation à Plovdiv 93.18\%(41), 4.55\% (2) a Sofia et 2.27\% (1) a Stara Zagora. À l'âge de 30 à 40 ans étaient 38 (86.36\%) médecins avec spécialité

Ce fait on explique par la présence de Faculté de médecine à l'Université de médecine de Plovdiv depuis la première moitié du siècle dernier.

La répartition des pédiatres et des médecins généralistes dans le service de santé est présenté dans le tableau 1 
Tableau 1. Répartition d'expérience des pédiatres et des médecins généralistes dans le service de santé

\begin{tabular}{|c|c|c|}
\hline & pédiatres & médecins généralistes \\
\hline De 5 ans & 3 & 3 \\
\hline De 6 a 10 ans & 5 & $\mathbf{6}$ \\
\hline De 11 a 20 ans & $\mathbf{1 1}$ & $\mathbf{9}$ \\
\hline Sur 20 ans & 4 & 3 \\
\hline & 23 & 21 \\
\hline
\end{tabular}

A 5 ans d'expérience ont 3 pédiatres et 3 médecins généralistes; 11 répondants ont une expérience de 6 à 10 ans. Le groupe le plus abondant est cet des répondants ayant une expérience de 11 à 20 ans 11- pédiatres et 9médecins généralistes. Plus de 20 années d'expérience ont 4 pédiatres et 3 médecins généralistes.

La majorité des répondants-17 pédiatres et 12 médecins généralistes ont acquis leur formation jusqu à 2000 années. Les autres 15 après 2000 .

Tous les répondants maintiennent le niveau de qualification par la formation postuniversitaire, ont des diplômes et des certificats, comme et spécialité reconnue.

La proportion de pédiatres - coordinateurs est 60.87\% (14), 8 sont stagiaires principaux et 1 est directeur Tous 21 médecins généralistes. La durée du service des répondants est présentée dans le tableau 2

Tableau 2 : Expérience de travail dans la santé

\begin{tabular}{|c|c|c|c|c|c|c|c|c|}
\hline & \multicolumn{2}{|c|}{ De 5 ans } & \multicolumn{2}{c|}{ De 6 à 10 ans } & \multicolumn{2}{c|}{ De 11 à 20 ans } & \multicolumn{2}{c|}{ Sur 20 ans } \\
\cline { 2 - 9 } & compte & $\%$ & compte & $\%$ & compte & $\%$ & compte & $\%$ \\
\hline pédiatres & 3 & 13.04 & 7 & 30.43 & 8 & 34.79 & 5 & 21.74 \\
\hline $\begin{array}{c}\text { médecins } \\
\text { généralistes }\end{array}$ & 5 & 23.81 & 6 & 28.57 & 3 & 14.29 & 7 & 33.33 \\
\hline
\end{tabular}

Les pédiatres ayant une expérience dans les soins de santé plus de 5 ans sont 20, seulement 3 ont une expérience de 5 ans. La répartition est identique et pour les médecins généralistes seulement 5 ont une expérience de 5 ans, et autres 16 ont une expérience dans les soins de santé plus de 5 ans.

En soins primaires travailler tous les GP-@@@@@@@@, en l'hôpital tous les pédiatres. Nous ne spécifions pas qu'ils travaillent dans les soins de santé publics ou privés, parce que nous sommes d'avis qu'il ne peut y avoir aucune différence significative dans la prescription de médicaments.

Notre étude visait à examiner les antibiotiques les plus couramment prescrits aux enfants dans l'année.

Le choix de l'antibiotique reste une question difficile dans chaque cas. Depuis plusieurs décennies infectionists, bactériologistes et les pédiatres ajustent et adaptent la stratégie de l'antibiothérapie empirique. Et elle est 
différente en fonction de la région, en raison de l'épidémiologie et de la résistance aux antibiotiques qui ne sont pas les mêmes.

Le plus souvent, les médecins utilisent: Duomox

Pénicillines à large spectre: Amoxicillin, Amopen, Ospamox,

Céphalosporines de première génération: Ospexin, Cephalexin

Céphalosporines de deuxième génération: Cefaclor

Céphalosporines de troisième génération: Abricef, Cefotaxime, Rocephin, Fortum

Céphalosporines de quatrième génération: Maxipime Macrolides: Macropen

Aminoglicosides: Gentamicine, Amicacine

Ils peuvent être classés par des principes différents. Dans la figure 1. Nous présentons une classification pharmacologique générale des antibiotiques.

Classification selon le mécanisme d'action est presentée dans la figure 1.

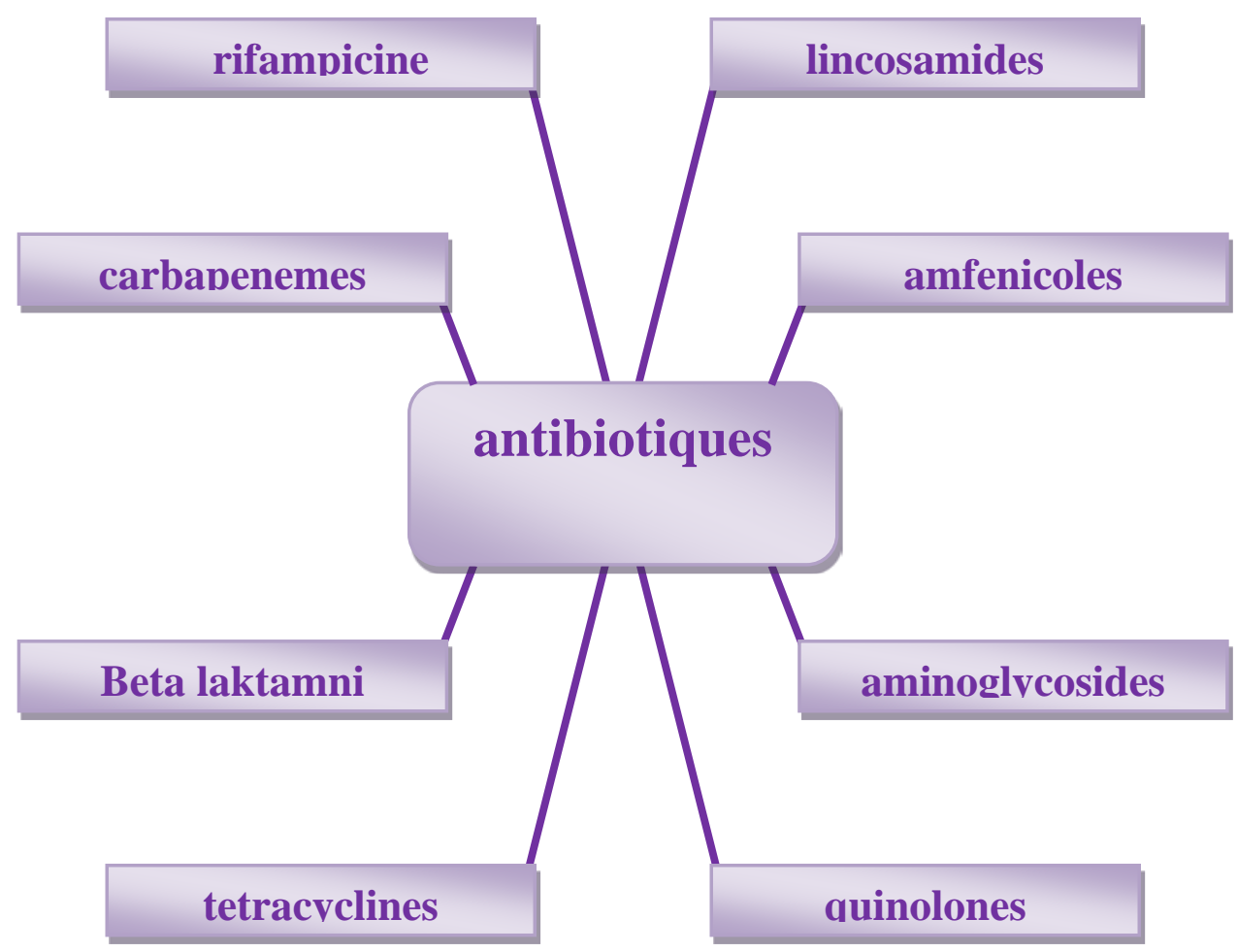

Figure 1. Classement général pharmacologique 
La classification d'antibiotiques selon le mécanisme d'action, la gamme et le type d'opération est présentée dans la figure 1.

Après le traitement des données personnelles sur la prescription la plus fréquente d'antibiotiques aux enfants, nous établions leur nombre moyen (Tableau 3)

Tableau 3: Nombre moyen d'emballages d'antibiotiques prescrites au cours de mois / année par les pédiatres pour les enfants.

\begin{tabular}{|c|c|c|}
\hline préparations antibiotiques & par mois, nombre & de l'année,nombre \\
\hline Cefzil 125/5 & 17 & 204 \\
\hline Cefzil 250/5 & 13 & 156 \\
\hline Azatril 100/5 & 5 & 60 \\
\hline Azatril 200/5 & 4 & 48 \\
\hline Pancef 60ml & 4 & 48 \\
\hline Pancef $100 \mathrm{ml}$ & 4 & 48 \\
\hline Ceroxim 125/5 & 5 & 60 \\
\hline Ceroxim 200/5 & 5 & 60 \\
\hline Cek & 2 & 24 \\
\hline Duracef 250/5 & 3 & 36 \\
\hline Tubocin & 2 & 24 \\
\hline Amikacin & 1 & 12 \\
\hline Ospamox 500mg/5ml & 1 & 15 \\
\hline Klacid $125 / 5$ & 2 & 24 \\
\hline Klacid 250/5 & 2 & 24 \\
\hline Medoklav 125/5 & 2 & 24 \\
\hline Medoklav 250/5 & 2 & 24 \\
\hline Medoclor 125/5 & 1 & 12 \\
\hline Medoclor 250/5 & 1 & 12 \\
\hline Gentamicin 2/40 & 8 & 96 \\
\hline Gentamicin 1/40 & 7 & 84 \\
\hline Gentamicin 1/80 & 5 & 60 \\
\hline
\end{tabular}

Analyse de la prescription de médicaments antibiotiques par pédiatres montre que le plus souvent ils prescrivent Cefzil 125/5 et Cefzil 250/5, environ 204 et 150 paquets par an. Son nom générique est Cefprozil. Cefzil est un semi-synthétique à large spectre céphalosporine antibiotique.

Troisième dans la pratique pédiatrique est Ceroxim 125/5-60 paquets par an prescrits et Ceroxim 250/5 aussi 60 paquets par an.

Également utilisé est Azatril 100mg / 5ml - 60 paquets. Et Azatril 250mg/5ml - 48 paquets. Son nom générique est Azithromycin. Azatril est un antibiotique macrolide dans le groupe des amides, nouvelle génération de macrolides. 
Inhibe la synthèse des protéines dans la cellule bactérienne et donne résultats dans le traitement de certaines infections.

Moins on prescript Pancef $60 \mathrm{mg} / 5 \mathrm{ml}$ et Pancef 100mg / 5ml - 48 paquets ou les deux types d'un total de 100 unités. Son nom générique est Cefixim. Pancefest est orale céphalosporine du groupe aminothiazole. Céfixime a montré une activité, à la fois in vitro et dans les infections cliniques contre la plupart des souches de micro-organismes suivants: Streptococcus influenza, Moraxella caterrhalis, Escherichia coli, Proteus mirabilis, Neisseria gonorrhoeae et d'autres.

Moins de Pancef est l'utilisation de Duracef $250 \mathrm{mg} / 5 \mathrm{ml}$ - environ 36-40 paquets par an. Son nom générique est Cefadroxil. Duracef est contreindiqué chez les patients présentant une allergie au groupe cephalosporin d'antibiotiques, ce qui est la raison probable pour l'application plus petite.

Klacid de $125 \mathrm{mg} / 5 \mathrm{ml}$ et de $250 \mathrm{mg} / 5 \mathrm{ml}$ ont écrit en 24 ou un total de 48 paquets par an. Son nom générique est Clarithromycin. klacid est un antibiotique appartenant au groupe appelé macrolides. Les antibiotiques macrolides arrêtont la croissance des bactéries qui causent des infections.

Telle est l'application de CEC forte 250mg / $5 \mathrm{ml}$ (24 paquets par an), avec nom générique Cefaclor. CEC forte est un antibiotique appartenant au groupe des céphalosporines, qui sont utilisés pour traiter les infections bactériennes. Il est utilisé pour traiter des infections aiguës et chroniques de la gravité differente, causées par des bactéries et aussi les maladies sont appropriées pour la thérapie par voie orale.

A 24 pièces d'emballage $125 \mathrm{mg} / 5 \mathrm{ml}$ et $250 \mathrm{mg} / 5 \mathrm{ml}$ est prescrit et Medoklav, qui est un antibiotique et travaille en tuant les bactéries qui causent des infections. Il contient deux substances différentes connues sous le nom de l'Amoxicilline et de l'Acide clavulanique. L'Amoxicilline est du groupe de médicaments connus sous le nom "Pénicilline" dont l'action peut parfois être entravée par des certains micro-organismes. L'autre composant actif - Acide clavulanique empêche que cela se produise. Amoxacilline + Acide clavulanique est la désignation générique.

L'application de Ospamox 500mg / 5ml est de 15 paquets par an. Son nom générique est Amoxacilline. Il contient de l'Amoxicilline. C' est un antibiotique qui tue les différents types de bactéries en perturbant la structure de la paroi cellulaire et par consequent est un agent approprié contre diverses infections.

Amikacin-12 par an.

Medoclor $125 \mathrm{mg} / 5 \mathrm{ml}$ et Medoclor forte $250 \mathrm{mg} / 5 \mathrm{ml}$ a été prescrit 12 fois au cours de l'année d'étude. Son nom générique est Cefaclor. Il appartie au groupe de médicaments appelés antibiotiques céphalosporines. Cefaclor acte en détruisant certaines bactéries causant l'infection. 
Les résultats et l'analyse des antibiotiques les plus couramment prescrits par les médecins généralistes présentont à la table. 4

Tableau 4 : Nombre moyen d'emballages d'antibiotiques prescrites au cours de mois / année par les pédiatres pour les enfants par GPs

\begin{tabular}{|c|c|c|}
\hline préparations antibiotiques & par mois, nombre & de l'année,nombre \\
\hline Ceroxim $125 / 5$ & 5 & 60 \\
\hline Zinnat & 15 & 180 \\
\hline Pancef & 2 & 24 \\
\hline Azatril & 15 & 180 \\
\hline Summamed & 5 & 60 \\
\hline Amikacin & 5 & 60 \\
\hline Ciprofloxacin & 2 & 24 \\
\hline Augmentin & 15 & 180 \\
\hline Amoxiclav & 5 & 60 \\
\hline Klacid & 10 & 120 \\
\hline Sumetrolim & 5 & 60 \\
\hline
\end{tabular}

Regardant les résultats transformés pour les antibiotiques les plus couramment prescrits par les médecins GPs, nous voyons l'utilisation la plus courante de Zinnat, Augmentin ES et Azatril. Azatril est un antibiotique macrolide appartenant au groupe des azatrils

Le représentant du groupe Macrolides Klacid (Clarithromycine) est prescrit 120 fois par an, alors l'ordre sont Sumamed, Amikacine - qui est un aminoglycoside et Amoxiclav d'environ 60 pièces par an. Aussi environ 60 pièces par an est prescrit et Sumetrolim.

Au moins dans leur pratique les médecins GPs sont utilisé Pantsef (Cefixime).

Ceci est céphalosporine de troisième génération et Ciprofloxacine - à environ 24 pièces par an, soit 1-2 par mois.

L'étude menée auprès de 32 pharmaciens de maître et 28 pharmaciens adjoints sur les antibiotiques les plus vendus pour les enfants généralisont au Tableau 5.

Tableau 5 Antibiotiques les plus souvent vendus pour les enfants

\begin{tabular}{|c|c|c|c|c|c|}
\hline $\begin{array}{c}\text { antibiotique } \\
\text { spécialite }\end{array}$ & $\begin{array}{c}\text { Cefzil } \\
125 / 5\end{array}$ & $\begin{array}{c}\text { Cefzil } \\
250 / 5\end{array}$ & Zinnat & Azatril & Augmentin \\
\hline Pharmaciens Master & 11 & 9 & 12 & 8 & 10 \\
\hline Assistant pharmacien & 6 & 4 & 3 & 7 & 5 \\
\hline total & 17 & 13 & 15 & 15 & 15 \\
\hline
\end{tabular}

Antibiotiques accordés par pharmaciens sont 30, et par assistants pharmaciens - 26.

\section{Conclusion}

Une lecture attentive des données de la littérature et de ce qui précède nous donne aux conclusions suivantes:

1. Aucune différence significative dans la prescription d'antibiotiques par les médecins généralistes et les pédiatres. 
2. Il n'y a pas de différence significative entre la prescription et les antibiotiques qui sont accordés, qui est un indicateur important qu' il n'y a pas de auto-guérison. Par conséquent, les patients comptent sur les experts médicaux.

\section{References:}

1. Guide pratique pour les étudiants de pédiatrie, les pédiatres et les médecins généralistes sous la redaction de $\mathrm{Dr} \mathrm{D}$. Petkov, $\mathrm{PhD}$ et professeur Z.. Dr Stanchev, II édition, Assoc. Zagora - Sofia, 2010

2. Krushkov I., L. Lambev S. Krushkova. (2006). Pharmacologie, sixième révision majeure, Sofia, Medical Publishing House "Arso"

3. Krushkov I., M. Markov, T. Traykov. (1988). Pharmacologie pour les techniciens de laboratoire médical et techniciens en radiologie, Sofia "Venimeks"

4. Médical standard pédiatrie, journal de l etat du 20/02/2011

5. Pédiatrie Un manuel pour les étudiants dans les programmes de premier cycle pour les professionnels médicaux et des activités sociales sous la redaction de Dr. G. Petkov, ST. Zagora, 2009

6. Pharmacologie sous la redaction de Professeur M. Karaivanova

7. Principes de pharmacologie médicale, sous la redaction de Ivan Lambev, éd. "Honey. et la gymnastique », Sofia, 2006

8. Беге П. и други. (1996). Практическа педиатрия, изд. ИК Парнас

9. Мумджиев Н. (2001). (2001) Основи на педиатрията. София, Медицинско издателство АРСО 